\title{
LOS MENORES Y LOS MAYORES: IMÁGENES INVERTIDAS DE LAS DESIGUALDADES POR EDAD
}

\author{
DANIEL LA PARRA CASADO \\ Departamento de Sociología II, Psicología, Comunicación y Didáctica. \\ Universidad de Alicante.
}

\section{RESUMEN}

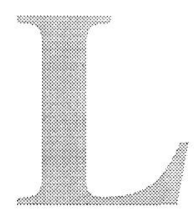

os procesos sociales de construcción de la infancia y la senectud pueden ser resultado de un mismo grupo de factores sociales (demográficos, económicos, ideológicos). En el artículo se analiza el modo en que se ha definido la participación en la sociedad de los menores y los mayores por parte de instituciones sociales como el Estado, el mercado de trabajo y los hogares. Se exploran las consecuencias en niños y mayores de la "exclusión" del mercado de trabajo, del acceso a los recursos sociales a través de las redes de solidaridad familiar y las implicaciones de las políticas sociales dirigidas a los menores y los mayores. Se observan los efectos de la definición social de la infancia y la senectud en la distribución de la renta, las oportunidades educativas y la atención a la salud dentro de estos grupos de edad. Se extrae la conclusión de que la representación de los menores y los mayores como "excluidos" del mercado laboral y protegidos por las relaciones de parentesco determina el tipo de soluciones que la política social y económica plantea para estos colectivos.

\section{INTRODUCCIÓN}

Para comprender las líneas principales que orientan la forma de plantearnos los problemas que afectan al mundo de los menores y los mayores, propongo introducir una especie de juego de espejos. Lo que pretendo es relacionar los procesos de construcción social de la imagen de los menores y los mayores estructurándolos dentro de una misma lógica. El objetivo es que al final de esta propuesta sean más visibles los aspectos esenciales que guían la problematización de la vida de 
los mayores y los menores. Cuando utilizo el vocablo problematización me refiero al hecho concreto de que nuestra forma de plantearnos los problemas está guiada por las representaciones generales que nos hacemos sobre el modelo de vida que deben tener las personas mayores (aquéllos a los que se suele llamar ancianos, tercera edad o mayores de 65 años) y los que son menores de edad (niños, infancia, adolescentes, menores de 18 años...). El interés de conocer la influencia de las representaciones culturales en los procesos de problematización es que éstas pueden estar guiando las políticas sociales con respecto a estos grupos de edad. Conocer dichas representaciones puede servir por tanto para adquirir autoconciencia sobre decisiones que por el momento se toman de forma automática por parte de cualquiera de los actores participantes en la intervención social (desde el trabajador social, hasta el Ministerio de Trabajo y Asuntos Sociales). Una mayor autoconciencia puede proporcionar las bases para un trabajo de autocrítica y el desarrollo de iniciativas más innovadoras. Por otro lado, la descripción de los procesos que están detrás de la construcción de nuestras ideas de infancia y senectud pueden servir para denunciar los principales mecanismos de creación de desigualdades por edad que afectan a estos grupos.

Habitualmente se presentan los procesos sociales que dieron lugar a las representaciones que actualmente compartimos sobre los menores y los mayores como procesos paralelos sin ningún tipo de conexión entre ellos. No obstante, la idea que aquí se defiende es que ambos procesos son resultado de una misma lógica y que en realidad ambos están intrínsecamente relacionados. Para argumentar esta idea se procederá a describir las principales características del proceso de construcción social de la imagen de los menores, para buscar posteriormente su reflejo en la construcción de la imagen de los mayores y, por último, tratar de esclarecer el tipo de lógica que explica la simetría entre ambas. Se avanza, no obstante, que dicha lógica tiene que ver con el modo en que la participación de la sociedad de los diferentes tipos de ciudadanos ha sido definida por parte de instituciones sociales como el Estado, el mercado de trabajo y los hogares.

\section{LA CONSTRUCCIÓN SOCIAL DE LA INFANCIA}

Si seguimos la propuesta (ya clásica) del historiador francés Ariès (1973), la idea de infancia no siempre estuvo presente en todas las sociedades. Más concretamente tal y como el autor argumenta los sentimientos actuales hacia la infancia no existían en el Antiguo Régimen. Éstos se fueron desarrollando paulatinamente durante la moder- 
nidad a medida que se va construyendo una nueva sociedad que implica todo tipo de cambios sociales provocados por procesos tales como la industrialización y la urbanización. Entre ellos cabe destacar, por su relevancia en la construcción social de la infancia, los cambios en las formas de vida familiar y más concretamente el origen de la familia nuclear (formada por el esposo, la esposa y los hijos que constituyen un grupo primario). Esta innovación crea el espacio social necesario (la vivienda en el ámbito urbano) para desarrollar una idea de la infancia ligada a la responsabilidad paterna y sobre todo materna (frente a las abundantes fuentes de sociabilidad de las que disponía un niño en las sociedades agrícolas del antiguo régimen) y centrada en la idea de protección del niño (frente a la indiferenciación entre niños y adultos en el trabajo y en el trato propia de la explotaciones agrícolas familiares del antiguo régimen).

En efecto, tal y como Rodríguez Pascual (2000) plantea en un artículo de revisión de estudios sobre la génesis del estatus del menor, los diferentes autores coinciden en señalar que revoluciones burguesa e industrial transformaron la realidad social creando toda una serie de cambios que confluyeron en la formación de la idea de infancia tal y como hoy la conocemos. Por un lado encontramos que se desencadena un cambio demográfico de gran magnitud. Como resultado, la esperanza de vida crece de forma continua de manera que existen más años en la vida de una persona. De esperanzas de vida al nacer entre los treinta y cuarenta años, se llega a las actuales esperanzas de vida de más de setenta años, que ofrecen una amplitud vital necesaria para la creación de etapas como la infancia, la adolescencia o la juventud. La transformación demográfica está totalmente relacionada con una transformación social en el papel jugado por los niños en la economía doméstica. Tal y como Kingsley Davis se encargó de demostrar los niños en un contexto de baja mortalidad y de transformaciones en la economía (el trabajo empieza a desarrollarse de forma asalariada y no lo organiza la unidad doméstica lo que hace que se retrase el inicio de la edad productiva del niño) suponen un coste creciente para los hogares, además de que una amplia descendencia provoca problemas de competencia por los recursos. Así en países como Francia los hogares que reducen el número de hijos aumentan sus probabilidad de movilidad social ascendente (Iglesias de Ussel, 1998). Todo ello lleva a cambiar la consideración del niño, quien pasa de ser tratado como un bien de producción a ser visto como un bien de consumo. El niño, alejado temporalmente de los procesos productivos, se empezará a ocupar en una nueva actividad: la educación.

De hecho, además de los cambios demográficos y económicos se han de destacar cambios culturales, sobre todo los que se enmarcan 
dentro del movimiento ilustrado, que dan lugar a obras como L'Émile (1762) de Jean-Jacques Rousseau, quien desde el naturalismo pedagógico se ocupa de la educación del ser humano desde que nace hasta que se instala plena y activamente en la comunidad mediante el trabajo y la formación de una familia. El siglo XIX y XX, según los países, han sido los siglos de la expansión del sistema educativo. Dicha expansión responde tanto a la extensión de las ideas ilustradas como sobre todo a las nuevas necesidades propias de la sociedad industrial. A medida que la industrialización avanza las necesidades educativas aumentan. Para las clases dirigentes porque la técnica, la legislación y las fórmulas aplicadas en la organización de la actividad industrial y comercial son cada vez más complejas y sólo pueden ser aprendidas dentro del sistema educativo. Para las clases obreras, porque las sociedades industriales necesitan de una mano de obra cualificada, alfabetizada y, por supuesto, disciplinada y obediente (dedicada y responsable en el lenguaje de las ofertas de empleo). El curriculum escolar de los niños de las clases populares estará impregnado a lo largo de este período de contenidos destinados a convertir al niño en un trabajador disciplinado tanto por los contenidos explícitos, como por los implícitos y por los universos simbólicos a través de los que se suele transmitir este conocimiento. Las ideas nacionalistas y religiosas fueron de gran utilidad a la hora de ofrecer un marco en el que situar esta nueva pedagogía. Ya en Rousseau se encuentra la idea de la utilidad pública de la educación (instalar al niño en la comunidad a través del trabajo y la formación de una familia). Desde un primer momento la educación obligatoria estuvo orientada a la inserción laboral y a los contenidos de tipo moralista. Aún hoy se entiende que la principal función del estudio es conseguir una mejor inserción laboral. La idea de que el sistema educativo pudiera servir para el desarrollo de la capacidad creativa, del sentido crítico o la autonomía de los individuos quedaba en un segundo lugar e incluso podía ser directamente perseguida. No se buscaba producir artistas, políticos o ciudadanos reivindicativos, sino honrados trabajadores.

La expansión del sistema educativo es una de las claves para comprender nuestro modelo actual de infancia. Se trata de una edad de la vida en la que el niño recibe de las generaciones adultas su formación. En la que tanto el hogar como el Estado han de encargarse de garantizar esa transmisión de conocimientos (a través de fórmulas como la escolarización gratuita y la prohibición del trabajo de los menores). La idea principal detrás de este planteamiento es que el niño ha de formarse para garantizar su inserción profesional. La desviación de esta norma (el fracaso escolar) se considera un aspecto negativo para el niño.

Dentro del sistema educativo las opciones no fueron, ni son iguales para todas las clases sociales. Por un lado se destaca la distinción 
entre escuelas de elite y escuelas populares, las cuales no siempre se corresponden con la división privada-pública. Por otro lado, la distinción entre el bachillerato y la universidad frente a la formación profesional, aunque dentro estos grupos también se producen interesantes elementos de estratificación.

La responsabilidad sobre la infancia aparecerá como resultado de todo este proceso ligada al hogar y a la escuela. Esta idea quedará incluso sancionada en las ciencias sociales por autores como Durkheim o Parsons quienes sitúan al hogar junto a la escuela como principales instancias en el proceso socializador, pero también penetra el conjunto de las ciencias que se desarrollan para atender a la infancia. La psicología, la pedagogía y hasta la pediatría han reforzado la idea de la responsabilidad paterna sobre los niños.

\section{LA CONSTRUCCIÓN DE LA REPRESENTACIÓN SOCIAL DE LOS MAYORES COMO IMAGEN INVERTIDA DE LA INFANCIA}

Entre las características básicas de la senectud (en el sentido positivo del término) se suele coincidir en señalar que en esta edad de la vida se produce un cambio en la posición de las personas en la actividad económica (la jubilación), que existen cambios en las relaciones familiares (los mayores pierden progresivamente su posición central con respecto a los hijos, ya sea porque éstos se emancipan o porque son los hijos quienes empiezan a organizar la vida en la unidad doméstica) reforzados en ocasiones por cambios en el estatus marital (viudedad). También puede ser representada como una edad en la que se deteriora de forma progresiva el estado de salud.

Si bien esta descripción repite en muy pocos puntos los señalados para la infancia, existen algunas coincidencias evidentes. Una de ellas es el hecho de que para que haya surgido la idea de que existe una edad tras la etapa adulta es necesario que la esperanza de vida sea mayor. También destaca que esta edad de la vida se caracteriza, al igual que la infancia, por la inactividad (laboral) y que ello ha sido posible, en los países industrializados, gracias a que la economía ha sido lo suficientemente productiva como para mantener a estos colectivos.

Más allá de estas coincidencias básicas en las formas, puede haber una serie de coincidencias más profundas en nuestra forma de representarnos a estos grupos de edad. Una de las primeras coincidencias tiene que ver precisamente con utilizar la edad como criterio clasificatorio. La edad sirve como una variable estructuradora de las oportuni- 
dades, capacidades y recursos con las que cuentan las personas. Esta forma moderna de estratificación social tiene que ver fundamentalmente con la posibilidad de participar en el mercado de trabajo, siendo el Estado el que actúa como principal actor a la hora de sancionar los límites inferior y superior de edad en los que se puede trabajar. No obstante, es destacable que las propias empresas podrán alterar dichos límites bien decidiendo contratar gente con una cierta experiencia profesional (esto es, gente con más edad del mínimo exigido para poder trabajar), bien cuando toman la opción de jubilar a despedir a los trabajadores mayores de una determinada edad. Es cierto que el proceso de estratificación por edad es mucho más intenso en los menores que en los mayores. Los niños llegan hasta el punto de ser clasificados por su año de nacimiento dentro del sistema educativo, pero lo relevante para la descripción que aquí se realiza es la constatación de que la edad pueda servir como un criterio para definir el acceso al mercado de trabajo para los mayores y los menores.

Parece por tanto que estos dos colectivos están excluidos del mercado laboral, sin embargo, lo cierto es que tanto los ancianos como niños acaban siendo clasificados en función de su relación con el trabajo. En los menores lo que se convierte en determinante es su trayectoria educativa dentro del sistema educativo. Se distingue entre aquéllos que han fracasado en el sistema escolar y los que no lo hacen. Dentro de los que siguen una trayectoria educativa exitosa se preparan para diferentes tipos de inserción profesional. Se les clasifica por tanto de forma anticipada en función de lo que podrán llegar a ser en el mundo laboral. Una situación totalmente invertida se encuentra en el caso de las personas mayores. Para ellos lo relevante no son ya la expectativas laborales, sino su historia laboral. El sistema de prestaciones sociales distingue entre aquéllos que han sido trabajadores activos (y entre ellos premia a los que estuvieron en mejores puestos de trabajo) y los inactivos (con derecho únicamente a pensión de tipo no contributivo). El equivalente al niño que fracasa en el sistema escolar es la persona mayor que por haber dedicado su vida a trabajos no mercantiles (tareas domésticas) e, incluso, esporádicamente a trabajos productivos, no llegan a contribuir lo suficiente como para percibir una pensión contributiva, sino una de una cuantía económica muy inferior (de tipo no contributivo).

Mayores y menores están estratificados en relación con el mercado de trabajo pero no participan directamente de él. La participación en el consumo de estos colectivos puede producirse por dos mecanismos principales. El primero (el predominante en los niños) es su integración en una familia, el segundo (válido para las personas mayores que han trabajado) las prestaciones por jubilación. En el caso de las 
personas mayores que no han trabajado se prevé una ayuda económica de marcado carácter asistencialista (la pensión no contributiva) y también la integración en el hogar. Esta dependencia de otros familiares de las personas ancianas (sobre todo mujeres) está de hecho reconocida en la llamada pensión de viudedad. Ésta se percibe en virtud de la relación de parentesco que une al beneficiario con alguien que ha fallecido. Esto es, el Estado sustituye la función económica dejada por el desaparecido "cabeza de familia", exactamente igual que en el caso de las pensiones de orfandad.

La responsabilidad de la institución familiar será por tanto la de integrar en los procesos de consumo. No obstante, una segunda responsabilidad para las familias se dibuja incluso con mayor fuerza. El hogar debe añadir dos elementos esenciales para la atención de los menores y los mayores (éstos últimos sólo cuando tienen problemas de salud): un volumen considerable de trabajo no mercantil y mecanismos de apoyo social informal. Se trata de garantizar la seguridad, las atenciones (limpieza, comidas, higiene) y el entorno afectivo necesarios para que el niño pueda crecer o los cuidados de salud que necesita una persona cuando padece alguna enfermedad de tipo crónico. Esta responsabilidad de los hogares (en especial de las mujeres) sobre los menores y los mayores está estrechamente vinculada a las transformaciones en las formas familiares que están detrás del origen de la infancia.

El desarrollo histórico de la idea de infancia se relaciona con la aparición y definición del rol materno. El desarrollo del modelo actual de infancia consolida nuevas funciones para las mujeres. A lo largo del proceso sumarán a sus anteriores obligaciones en la economía doméstica, mayores responsabilidades en la crianza de los hijos (mucho más allá de las funciones meramente biológicas) y sobre todo en la educación y protección de los mismos. Todo ello sin que se haya alterado su situación subordinada con respecto al marido dentro del hogar. Se llega incluso, sobre todo en la segunda mitad de este siglo, a que incorporen incluso obligaciones como trabajadoras asalariadas.

De forma análoga a lo que ocurre en la infancia, se encuentra que si las personas mayores se convierten en personas dependientes debido al deterioro de la capacidad física o cognitiva también se esperará que sea la familia la que resuelva sus problemas. Esta labor se realizará de forma prioritaria por parte del propio cónyuge de la persona anciana con problemas de salud (sobre todo cuando es mujer) o por parte de las hijas de la persona anciana.

Se ha argumentado que el mercado de trabajo y las relaciones de parentesco actúan como nexo de unión entre las situaciones vividas 
por los menores y los mayores. Todavía se puede encontrar un tercer nexo en la forma en que el Estado plantea su política social en favor de estos colectivos. En este caso la definición predominante por parte de la política pública hacia estos colectivos se ha centrado en la idea de que son dependientes. En el caso de los niños la idea de dependencia se materializa sobre todo en la consideración de la infancia como una edad a proteger. En los casos como el español en los que las iniciativas en política familiar son tan limitadas (Iglesias de Ussel, 1998) esta orientación es perceptible en el desarrollo legislativo, en actuaciones concretas como la retirada de la tutela de los hijos o la persecución del maltrato infantil o en el establecimiento de ayudas económicas en favor de los menores (becas escolares, ayudas económicas por hijo o familia numerosa). Esta idea de dependencia se encuentra de forma en el caso de las personas mayores, tal y como argumenta Guillemard (1992) al analizar las políticas de vejez aplicadas en Europa tras la II Guerra Mundial, se ha convertido a lo que se ha llamado "Tercera Edad" en una edad dependiente. La autora señala que las iniciativas políticas se han centrado en aspectos tales como la institucionalización de las personas mayores o su medicalización.

Otros aspectos culturales unen las representaciones sociales de la infancia y la senectud. Algunos de ellos pueden parecer meramente anecdóticos o simplemente lógicos, pero en cualquier caso revelan la fuerza de las representaciones sociales. Estas edades de la vida presentan por ejemplo muy bajos niveles de nupcialidad. En el caso de los menores simplemente porque los matrimonios están prohibidos, pero en el caso de los mayores la prohibición legal no actúa. Son excepcionales los célibes, divorciados y viudos que a estas edades de la vida deciden unirse en matrimonio. De igual modo es llamativo que el celibato propio de los menores se extienda entre los más jóvenes quienes retrasan su edad hasta los treinta años. Quizá todos estos fenómenos tengan que ver con las responsabilidades que los actores sociales estamos asignando a los matrimonios en edad de procreación.

De igual modo se pueden destacar la semejanza entre las pautas de sociabilidad de los niños y los ancianos. Sobre todo en la primera infancia el círculo de relaciones sociales se limita a su círculo familiar. Tal y como señalaba Ariès esta pauta de sociabilidad en la infancia es típica de nuestras sociedades urbanizadas (e industrializadas) en las que la vida familiar dentro del hogar adquiere una especial densidad. De forma análoga en las personas mayores, la desvinculación del trabajo productivo puede suponer una reducción en los niveles de sociabilidad, que suelen verse muy afectados sobre todo en caso de enfermedad crónica hasta reducirse únicamente a las relaciones familiares. Por supuesto esto no ocurre en todos los casos, más aún cuando las perso- 
nas mayores no padecen procesos de enfermedad o cuando cuentan con otras fuentes de sociabilidad (por ejemplo, la de los vecinos, aunque esta es más fácil en el ámbito rural) o con redes de amistades que no estaban vinculadas al trabajo, sino al consumo cultural (lo que es más propio de clases sociales con altos niveles adquisitivos) (Due, 1999; Matthews, 1999; Requena, 1991 y 1996; Rosengren, 1993 y Stansfeld, 1999).

Después de la enumeración de las coincidencias existe al menos un punto en el que el tratamiento de la infancia y la vejez son claramente diferentes. En el campo de la educación se dibuja una diferencia fundamental puesto que mientras la infancia está marcada por la institución escolar apenas existen intentos de insertar a la población mayor en los procesos formativos (con excepciones como la Universidad Permanente en la Universidad de Alicante destinada a las personas mayores). En ocasiones incluso se han transmitido ideas sobre la incapacidad para aprender en esta edad. La falta de insistencia en la formación parece más relacionada con el valor laboral que se ha concedido a la educación por encima de otros valores como pueda ser la de desarrollo personal.

\section{REPERCUSIONES SOCIALES DE LAS REPRESENTACIO- NES SOCIALES DE LOS MENORES Y LOS MAYORES}

Una de las consecuencias de la definición de la infancia y la senectud como edades dependientes, y sobre todo, de su exclusión del mercado de trabajo, es que dependen de los sistemas de solidaridad que se establezcan para participar del consumo. Esto tiene importantes consecuencias. Las estadísticas sobre pobreza muestran de forma repetida que los niños y los ancianos tienen mayor probabilidad de situarse bajo la línea de pobreza (definida como la mitad de la mediana del ingreso disponible por hogar) en nuestro país y en la mayoría de los países de nuestro entorno (Smeeding, 1997). La situación de los menores y los mayores puede variar de unos países a otros en función de cómo se defina esta dependencia. En concreto en algunos países es la solidaridad familiar la que se supone que ha de atender las situaciones de los niños y ancianos, de manera que los ingresos de estos colectivos dependen de la redistribución en el interior de los hogares. En otros países sin embargo es el Estado mediante su política social el que se sitúa como primer responsable. De hecho es interesante observar como en algunos países no se cumple la norma de que los niños tengan una mayor vulnerabilidad frente a la pobreza. Uno de los casos más interesantes lo ofrece Finlandia, donde los hogares con niños tienen menor 
riesgo de estar bajo la línea de pobreza. Este hecho se puede relacionar con la existencia de toda una serie de subsidios universales (en forma de ayudas económicas y servicios sociales) destinados a la protección de la infancia y al estímulo de la incorporación de la mujer al trabajo (Gornick, 1997, 1998a y 1998b). Föster (1994) muestra que en países como Suecia, Holanda y el Reino Unido este tipo de ayudas suponen grandes reducciones en el índice Sen de pobreza de los hogares monoparentales. En Suecia este tipo de hogar llega a contar con niveles de pobreza inferiores a los de la media de la población. Por el contrario, en países como Estados Unidos, Australia y Canadá los impuestos y los subsidios sólo reducen muy ligeramente la pobreza de los hogares monoparentales. Este mismo autor ha mostrado que para 13 países de la OCDE existe una relación lineal entre el porcentaje que los gastos sociales suponen en el PIB y el porcentaje de hogares que se sitúan por debajo de la línea de pobreza. En el caso de los ancianos podemos encontrar que también se trata de un colectivo muy vulnerable frente a la pobreza (Rodríguez, 1994). En España la situación de los ancianos en los últimos años ha mejorado gracias a la consolidación de los sistemas de pensiones y a iniciativas como la posibilidad de acceder a pensiones no contributivas a partir de los 65 años, gracias a la reforma legal del año 1990 (Ley 26/1990). Es decir, la situación ha ido mejorando conforme el sistema ha pasado de la confianza en la solidaridad intergeneracional y en el salario familiar aportado por el marido, a un sistema que ha crecido de forma modesta en el reconocimiento de los derechos universales de las personas mayores.

TABLA 1. Porcentaje de hogares bajo la línea de pobreza.

\begin{tabular}{|l|c|c|c|c|c|}
\hline & Año & Todos & Mayores de 65 años & Adultos & Niños \\
\hline España & 1990 & 10,4 & 11,4 & 9,2 & 12,8 \\
\hline Finlandia & 1991 & 6,2 & 14,4 & 5,8 & 2,7 \\
\hline Noruega & 1991 & 6,6 & 13,5 & 5,4 & 4,9 \\
\hline Reino Unido & 1991 & 14,6 & 23,9 & 10,7 & 18,5 \\
\hline EE.UU. & 1994 & 19,1 & 19,6 & 16,4 & 24,9 \\
\hline
\end{tabular}

La línea de pobreza se establece en la mitad de la mediana del ingreso disponible por hogar ajustado por tamaño del hogar. El ajuste se realiza utilizando $E=0.5$, donde el ingreso ajustado es igual al ingreso total dividido por el tamaño del hogar elevado a E. Ingreso ajustado=ingreso total $/ S^{E}$. Elaboración a partir de datos del Luxembourg Income Study. Smeeding (1997).

Más allá de la riqueza de rentas, se puede observar el bienestar de los menores y los mayores en términos de capacidades. Entre ellas podemos seleccionar el caso de la educación para los menores y de la atención a la salud en el caso de los mayores. Si observamos las trayectorias en el sistema educativo de las niñas y niños españoles se 
puede comprobar que existen grandes diferencias en los niveles de realización escolar. En la tabla 2 se puede observar en qué medida el nivel de estudios del padre (el Instituto Nacional de Calidad y Evaluación no proporciona datos sobre la madre) determina las posibilidades educativas del niño. En concreto, mientras un $78 \%$ de las hijas de universitarios accede a la Universidad, sólo un $19 \%$ de las hijas de padres analfabetos o con estudios incompletos lo hace. Más interesante aún que este extraordinario nivel de desigualdad en la realización es el hecho de que mientras que un $85 \%$ de las hijas de universitarios que han completado los estudios que dan acceso a la Universidad finalmente acceden a la misma, entre las hijas de padres analfabetos sólo acceden un 55\% de las que podrían hacerlo. Evidentemente en esta elección no están incidiendo los niveles académicos, pues se cuenta con el nivel adecuado para acceder, sino otro tipo de condicionantes. Las causas, tal y como Boudon (2000) brillantemente ha explicado para el caso francés, se relacionan con el hecho de que los estudiantes toman esta

TABLA 2. Porcentaje de alumnos con posibilidad de acceder y que accede a la Universidad según el nivel de estudios del padre, 1987 y 1997.

Nivel de estudios del padre en 1987

\begin{tabular}{|c|c|c|c|c|c|}
\hline & & $\begin{array}{c}\text { Analfabetos } \\
\text { o con estudios } \\
\text { incompletos }\end{array}$ & $\begin{array}{l}\text { Estudios } \\
\text { primarios }\end{array}$ & $\begin{array}{c}\text { Estudios } \\
\text { medios }\end{array}$ & $\begin{array}{c}\text { Estudios } \\
\text { universitarios }\end{array}$ \\
\hline \multirow[t]{2}{*}{$\%$ con posibilidad de acceso ${ }^{\prime}$} & Varones & 13 & 34 & 67 & 82 \\
\hline & Mujeres & 20 & 45 & 78 & 88 \\
\hline \multirow[t]{2}{*}{$\%$ que accede a la Universidad } & Varones & 5 & 15 & 41 & 64 \\
\hline & Mujeres & 9 & 25 & 53 & 69 \\
\hline \multirow[t]{2}{*}{ Relación acceso-posibilidad } & Varones & 36 & 45 & 62 & 78 \\
\hline & Mujeres & 44 & 56 & 68 & 79 \\
\hline
\end{tabular}

Nivel de estudios del padre en 1997

\begin{tabular}{|c|c|c|c|c|c|}
\hline & & $\begin{array}{l}\text { Analfabetos } \\
\text { o con estudios } \\
\text { incompletos }\end{array}$ & $\begin{array}{l}\text { Estudios } \\
\text { primarios }\end{array}$ & $\begin{array}{c}\text { Estudios } \\
\text { medios }\end{array}$ & $\begin{array}{c}\text { Estudios } \\
\text { universitarios }\end{array}$ \\
\hline \multirow[t]{2}{*}{ \% con posibilidad de acceso ${ }^{I}$} & Varones & 21 & 42 & 68 & 87 \\
\hline & Mujeres & 34 & 58 & 82 & 91 \\
\hline \multirow{2}{*}{ \% que accede a la Universidad } & Varones & 9 & 21 & 43 & 67 \\
\hline & Mujeres & 19 & 34 & 60 & 78 \\
\hline \multirow[t]{2}{*}{ Relación acceso-posibilidad } & Varones & 45 & 50 & 64 & 76 \\
\hline & Mujeres & 55 & 58 & 73 & 85 \\
\hline
\end{tabular}

1. Con posibilidad de acceso: jóvenes de 19 a 23 años con el nivel de estudios requerido para acceder a la Universidad.

FUENTE: INCE, Sistema Estatal de Indicadores de la Educación, 2000. 
decisión haciendo una valoración de los costes económicos que supondría continuar con los estudios. La relación entre ventajas y costes es desfavorable en el caso de las hijas e hijos en familias con bajos ingresos y por ello renuncian a continuar los estudios incluso cuando ello sería posible. La necesidad de depender del hogar para las posibilidades educativas es la que determina el nivel de éxito en el sistema educativo, incluso una vez anulado el filtro de las calificaciones.

En el caso de la atención a la salud de las personas mayores podemos ver que se produce una situación similar en la medida que el nivel de atención que reciben también puede estar en relación con la posición socioeconómica del hogar en que se insertan. En el caso de las personas mayores con problemas de incapacidad física o psíquica la ayuda prestada por familiares es esencial para el mantenimiento de un determinado nivel de calidad de vida. Al menos así lo sugieren los datos de una encuesta estatal realizada por el INSERSO en 1995, según la cual las personas mayores de 65 años que necesitaban ayuda la recibían principalmente de familiares $(86,5 \%)$, en un $6,6 \%$ contratando empleados de hogar y en un $4,7 \%$ de los servicios sociales públicos (Instituto Nacional de Servicios Sociales, 1995). En este sentido tiene gran relevancia la pregunta sobre en qué medida puede variar el grado de apoyo familiar recibido en función del nivel socioeconómico del hogar en el que se vive. Ofrecer datos concretos sobre este particular supone una gran dificultad dada la escasez de fuentes estadísticas que aporten información en un mismo cuestionario sobre la persona cuidadora, la persona dependiente y que, a la vez, determinen con suficiente precisión el nivel socioeconómico del hogar. El Panel de Hogares de 1994 puede servir modestamente a este propósito en la medida que al tratarse de una encuesta de presupuestos familiares permite clasificar a los individuos según el nivel de ingresos del hogar (en ingresos por unidad de consumo del hogar); es posible a su vez identificar a las personas con mala salud (las que señalan tener alguna incapacidad que le limita intensamente en su actividad diaria o las que dicen tener mala o muy mala salud) y, por último, puesto que se pregunta al conjunto de miembros de cada hogar, se puede identificar en qué hogares hay una persona prestando cuidados de salud y en cuáles no.

A partir de esta información se ha construido la tabla que se incluye a continuación. En ella se observa que, excluyendo a las personas que prestan cuidados de salud, hay un $4,5 \%$ de personas que viven en hogares en los que nadie presta cuidados de salud y, sin embargo, dicen padecer alguna incapacidad que les condiciona intensamente en su actividad diaria (tabla 3). Este fenómeno es producido por el gradiente social en la prevalencia de las discapacidades, esto es, porque existe una mayor concentración de personas con un grado intenso de limitación en su 
actividad diaria entre las personas con menos ingresos (Navarro y Benach, 1996; Regidor, 1994), pero también puede relacionarse con la menor capacidad de los hogares con menos ingresos para movilizar la solidaridad familiar. El resultado es que la probabilidad de encontrarse con un problema de salud grave y sin cuidados en la clase social baja es mayor que en las clases sociales altas. En concreto, esto le ocurre al $5,2 \%$ de las personas entrevistadas con un bajo nivel de ingreso, frente al 2,8\% de las personas con un alto nivel de ingreso. En esta tabla no se desglosan los datos para varones y mujeres dado que el número de casos en alguna de las celdas era excesivamente reducido.

TABLA 3. Porcentaje de personas mayores de 65 años con un problema de salud que les limita intensamente en su actividad diaria y que viven en hogares en los que no hay ninguna persona prestando cuidados de salud. Panel Europeo de Hogares para España, 1994. PHOGUE 1994.

\begin{tabular}{|l|c|c|c|c|c|}
\hline \multicolumn{5}{|c|}{ Cuartil de ingresos del hogar } \\
\cline { 2 - 6 } & Alto & Medio-alto & Medio-bajo & Bajo & Total \\
\hline Total & $2,8 \%$ & $4,2 \%$ & $5,7 \%$ & $5,2 \%$ & $4,5 \%$ \\
\hline
\end{tabular}

FUENTE: fichero de microdatos PHOGUE (elaboración propia).

Los ejemplos sobre la distribución de la renta, las oportunidades educativas o el grado de acceso al apoyo de los familiares en caso de enfermedad pueden servir para ilustrar en qué medida los datos contradicen en algunos puntos las visiones habituales sobre el papel que juegan las familias en estas edades de la vida. En el discurso político (en las representaciones socialmente compartidas) es habitual escuchar que la renta se redistribuye hacia los niños a través de los padres, que las redes de solidaridad familiar se estrechan y se hacen más sólidas en las clases desfavorecidas (también denominadas trabajadoras, populares, obreras, pobres, excluidas en función de los lenguajes, las teorías y las ideologías) o que existe igualdad de oportunidades educativas. Además de los datos aquí aportados, las investigaciones sobre distribución de la renta, sobre el sistema educativo y, en menor medida, sobre la familia, se han encargado de desmentir cada uno de estos puntos o al menos los han matizado considerablemente, sin embargo, siguen pensando en la configuración de las políticas hacia los mayores y los menores.

\section{MENORES Y MAYORES: IMÁGENES INVERTIDAS PRO- YECTADAS SOBRE UN MISMO ESPEJO}

La infancia y la jubilación se presentan como excluidas del mercado de trabajo. La valoración de esta exclusión puede variar. En algunos 
casos se contempla como derechos adquiridos en esas edades de la vida (protección de la infancia, derecho al descanso y a la protección de la salud en la última edad de la vida), en otras casos la falta de participación en el mercado de trabajo se interpreta como una limitación injustificada de la libertad y de las oportunidades vitales (sobre todo en el caso de la jubilación). Con independencia de la valoración que cada uno haga de esta exclusión, como se ha argumentado, se puede discutir el que realmente estos colectivos estén excluidos del mercado laboral. Desde el inicio de la edad escolar y de forma mucho más evidente, desde el final de la escolarización obligatoria toda persona sigue una trayectoria educativa que va definiendo su identidad como trabajador (el tipo de oficio, o mejor dicho, de oficios que desempeñará), pero también y sobre todo el tipo de condiciones de trabajo a las que puede aspirar. Por su parte, para las personas mayores existe una relación directa entre su trayectoria laboral y la pensión que reciben, los planes de pensiones privados de los que algunos disfrutan, el seguro médico que les atiende o los niveles de servicios que les garantizan sus mutuas o la seguridad social. La relación es aún más evidente para las que no tuvieron una trayectoria laboral reconocida por las instituciones por dedicarse al trabajo doméstico o a actividades informales o interrumpidas por las obligaciones familiares, sobre todo mujeres. La idea de exclusión puede llevar a una percepción equivocada de las luchas de intereses que se enfrentan al abordar las políticas dirigidas a los menores y los mayores. Cuando se planifica una reforma del sistema educativo, cuando se alteran los sistemas de acceso a los diferentes tipos de estudio, cuando se deciden las fuentes de financiación para las escuelas públicas y privadas se están definiendo la estructura del mercado de trabajo en los próximos años. Así los grandes saltos cualitativos y cuantitativos entre los niveles de calidad de las enseñanzas profesionales y universitarias responden a la distinción ya clásica entre trabajos manuales y no manuales, la cual contribuyen a reforzar. De igual modo dentro de la enseñanza universitaria las diferencias entre las distintas carreras en los niveles de financiación, de dotación de infraestructuras y de profesorado (por ejemplo a través del reconocimiento de diferentes grados de experimentalidad), en el establecimiento de números límites de admisión, en la duración de los ciclos formativos o de posibilidades de obtención de becas de investigación son un reflejo de las diferentes posiciones ocupadas en el mercado de trabajo por las distintas profesiones. Las reformas educativas contribuyen a aumentar las diferencias o a disminuirlas, de ahí su importancia y el debate que suscitan.

Lo mismo ocurre en el caso de las personas mayores. Los debates sobre la política de pensiones no pueden entenderse sin referencia al 
mercado de trabajo. La alteración en las prestaciones económicas (la cuantía de las pensiones) o sociales (como el seguro de dependencia) recibidas por las personas mayores supone directamente una alteración de las condiciones del mercado de trabajo, de los sistemas de protección establecidos para los trabajadores, de las formas de contratación, de las obligaciones fiscales para trabajadores y empresas. De hecho esto es algo reconocido en la medida que se reconoce como interlocutores válidos en las mesas de negociación sobre pensiones a sindicatos y patronal.

Pero entonces, ¿por qué seguir hablando de exclusión del mercado de trabajo de los menores y los mayores?. Sin entrar en las motivaciones que puede haber detrás de la enunciación de una idea, sin duda variadas, el mantenimiento del pensamiento de que estos grupos de edad no están relacionados con el mercado de trabajo contribuye en poco a reducir las desigualdades que se producen en el interior de estos grupos (en niveles de consumo, en oportunidades educativas, en protección frente a la pobreza, en calidad de vida).

Cuando la conexión con el mercado de trabajo no es visible las soluciones a plantear para la resolución de los problemas que afectan a la infancia y a la senectud se reducen a la política social y a las posibilidades que ofrezca la solidaridad familiar.

En el plano ideológico una de las soluciones más frecuentemente planteadas para resolver los problemas de los menores y los mayores es reforzar, incentivar o mantener los actuales niveles de solidaridad familiar. En sus formas más acentuadas el familismo defendería que la reciprocidad familiar es la forma de extensión del bienestar y los beneficios obtenidos por los trabajadores hacia los niños, los ancianos y las mujeres (inactivas). Esta ideología se ve correspondida en países como España por una realidad empírica en la que se encuentran sólidos lazos familiares que actúan protegiendo a dichos colectivos. Desde esta perspectiva la política social cumpliría la función de cumplimentar los huecos no cubiertos por la solidaridad familiar. La casuística de grupos a proteger puede variar, pero normalmente tiende a incluir: niños en "familias desestructuradas", niños víctimas de la violencia, niños huérfanos, ancianos sin familia o las viudas. Las medidas que se arbitran tienen un carácter limitado: pensiones no contributivas de baja remuneración (en España en el año 2000 la cuantía era de 40.260 ptas/mes), acogimientos familiares, prever (un bajo número de) plazas en residencias públicas para ancianos sin familia (Pérez Díaz, 1998; Defensor del Pueblo, 1990).

Si bien se ha comentado que el familismo en el caso español cuenta con el apoyo de que en la realidad social española la familia proporcio- 
na una amplia red de solidaridad, no obstante puede obviar toda una serie de costes sociales y deficiencias de un sistema de bienestar que confía en los hogares. En primer lugar se puede destacar el amplio coste (a menudo invisible) que recae en las mujeres, sobre todo debido a cuestiones tales como la crianza de niños o la atención a personas mayores que suponen importantes limitaciones en sus oportunidades laborales, en su independencia económica y en su disponibilidad de tiempo (Durán, 1999). En segundo lugar, los hogares con menos ingresos tienen menos recursos para la movilización de la solidaridad familiar. Las clases sociales desfavorecidas cuentan por ello con menores oportunidades educativas para sus hijos, menor nivel de atención familiar cuando se es una persona mayor enferma o la necesidad de realizar mayores inversiones de tiempo a la hora de atender a familiares ancianos o enfermos (La Parra, 2001).

La visión de los menores y los mayores como grupos caracterizados por la edad ha contribuido a hacer menos visibles las desigualdades que se dan en el interior de estos colectivos. Dichas desigualdades están relacionadas, por un lado, con las particulares formas de adscripción de estos colectivos al mercado de trabajo. Por otro lado, se relacionan con las desiguales capacidades de los hogares, las familias si se prefiere, para proporcionar las mismas oportunidades a los niños, o los mismos niveles de atención a la salud a los mayores. Sin embargo, la existencia de estas amplias redes de solidaridad familiar en el caso español tienen el efecto de hacer invisibles las desigualdades que se derivan de fundamentar la participación en la sociedad de estos grupos de edad en las relaciones familiares. La política social puede contribuir a la reproducción de estos esquemas o a su sustitución por nuevas formas de definir la participación de las personas, más concretamente de los menores y los mayores, en la sociedad.

En el primer caso (el familista), como se ha visto, se definirán medidas políticas destinadas a cubrir los casos en los que la red familiar no cubre su "función". En un segundo caso, de carácter más universalista, según la clasificación de (Esping Andersen -1993-), las medidas adoptadas se entienden como derechos compartidos por todos los menores o los mayores, cualesquiera que sea su situación familiar, social o económica. Esta forma de protección propia de los países nórdicos europeos garantiza unos elevados niveles de calidad de vida, así como unas bajas tasas de pobreza en los menores y los mayores, y mayores oportunidades vitales para las mujeres. 


\section{BIBLIOGRAFÍA}

ARIÈS, P.: L'enfant et la vie familiale sous L'Ancian Régime. Seuil, París, 1973.

BOUDON, R., CUIN, CH. y MASSONT, A.: L'axiomatique de l'inégalité des chances. L'Harmattan, París, 2001.

CASTELLS, M. y ORTIZ, LP.: Análisis de las políticas de vejez en España en el contexto europeo. Ministerio de Asuntos Sociales, Instituto Nacional de Servicios Sociales, Madrid, 1992

DEFENSOR DEL PUEBLO: Residencias públicas y privadas de la Tercera Edad. Madrid, 1990.

DUE, P. et al.: "Social Relations: network, support and relational strain", Social Science and Medicine, 1999, 48: 661-673.

DURÁN, MA.: Los costes invisibles de la enfermedad. Fundación BBV, Madrid, 1999.

ESPING-ANDERSEN, G.: Los tres mundos del estado del bienestar. Alfons el Magnànim, Institució Valenciana d'Estudis i Investigació, Valencia, 1993.

FÖRSTER, MF.: "Measurement of low incomes and poverty in a perspective of international comparisons", OCDE Labour market and social policy occasional papers, 1994, n¹4, OCDE/GD (94) 10.

GORNICK, JC. y JACOBS, JA.: "Gender, the Welfare State, and Public Employment: A Comparative Study of Seven Industrialized Countries", American Sociological Review, 1998, 53: 688-710.

GORNICK, JC., MEYERS, MK. y ROSS, KE.: "Public policies and the Employment of Mothers: A Cross-National Study". Social Science Quaterly, 1998, 79:35-54.

GORNICK, JC., MEYERS, MK. y ROSS, KE.: "Supporting the employment of mothers: policy variation across fourteen welfare states", Journal of European Social Policy, 1997, 7: 45-70.

GUILLEMARD, AM.: Análisis de las políticas de vejez en Europa. Ministerio de Asuntos Sociales. Instituto Nacional de Servicios Sociales, Madrid, 1992.

IGLESIAS DE USSEL, J.: La familia y el cambio político en España. Tecnos, col. de Ciencias Sociales, Madrid, 1998.

INSTITUTO NACIONAL DE SERVICIOS SOCIALES: Cuidados en la vejez. El apoyo informal. Ministerio de Asuntos Sociales, Madrid, 1996.

LA PARRA CASADO, D.: "Contribución de las mujeres y de los hogares más pobres a las producción de cuidados de salud informales", Gaceta Sanitaria, 2001, 15: 498-505.

MATTHEWS, S., STANSFELD, S. y POWER, C.: "Social support at 33: the 
influence of gender, employment status and social class", Social Science and Medicine, 1999, 49: 133-142.

NAVARRO, V. y BENACH, J.: "Informe de la Comisión Científica de Estudios de las Desigualdades Sociales en Salud en España", Revista de Salud Pública, 1996, 70: 505-636.

PÉREZ DÍAZ, C., CHULIÁ, E., y ÁLVAREZ MIRANDA, E.: Familia y sistema de bienestar. La experiencia española en el paro, las pensiones, la sanidad y la educación. Argentaria, Madrid, 1998.

REGIDOR, E., GUTIÉRREZ-FISAC, JL. y RODRÍGUEZ, C.: Diferencias y desigualdades en salud en España. Díaz de Santos, Madrid, 1994.

REQUENA SANTOS, F.: Redes sociales y cuestionarios. CIS, Cuadernos metodológicos, Madrid, 1996.

REQUENA SANTOS, F.: Redes sociales y mercado de trabajo. CIS/ Siglo XXI, Madrid, 1991.

RODRÍGUEZ PASCUAL, I.: "¿Sociología de la infancia? Aproximaciones a un campo de estudio difuso". Revista Internacional de Sociología, 2000, 26: 99-124.

RODRÍGUEZ, JA.: Envejecimiento y familia. CIS, Madrid, 1994.

ROSENGREN, A., ORTH-GOMER, K., WEDEL, H. y WILHELMSEN, L.: "Stressful life events, social support, and mortality in men born in 1933". British Medical Journal, 1993, 307: 1102-105.

SMEEDING, TM.: "Financial Poverty in Developed Countries: the Evidence From the Luxembourg Income Study", Poverty and Human Development, Human Development Report Office, the United Nations Development Programme, New York, 1997.

STANSFELD, SA.: "Social support and social cohesion", en Marmot, M. y Wilkinson, RG. (eds.) Social Determinants of Health. Oxford University Press, New York, 1999. 